\title{
El ingreso a carreras de alta o baja demanda en una universidad mexicana: ¿qué influye en la elección?
} The Access to Degrees of High or Low Demand in a Mexican University: What Influences the Election?

\section{Mónica López Ramírez}

\section{Resumen}

En este artículo se analiza la elección por una carrera de alta o baja demanda entre estudiantes de una de las principales universidades públicas mexicanas. Por un lado, se realiza una caracterización de una muestra de 1370 estudiantes a su ingreso a la universidad, contrastando de acuerdo con los resultados académicos obtenidos en el nivel educativo previo. Por otro, se analiza la incidencia de las condiciones sociales y culturales de origen de los estudiantes, así como su trayectoria académica previa en las probabilidades de elegir una carrera de alta y baja demanda en la universidad. Los resultados muestran que las mujeres, estudiantes mayores a 19 años, con padres que cuenten con educación superior, que hayan concluido el nivel educativo previo en el tiempo reglamentado, con promedios altos y que provengan del sistema de bachillerato de la UNAM, tienen mayores probabilidades de elegir carreras de alta demanda.

\section{Palabras claves}

Estudiantes, elecciones educativas, origen social, trayectoria escolar, México.

\section{Abstract}

This article analyzes the election for a career of high or low demand among students of one of the main Mexican public universities. On the one hand, a characterization of a sample of 1370 students upon entering the university is carried out, contrasting with the academic results obtained in the previous educational level. On the other hand, the incidence of students' social and cultural conditions of origin is analyzed, as well as their previous academic paths in the probabilities of choosing a career of high and low demand in the university. The results show that women, students over 19 years old, with parents who have higher education, who have completed the previous educational level in the regulated time, with high averages and who come from the baccalaureate system of the UNAM, are more likely to choose high demand careers.

\section{Keywords}

Students, educational choices, social background, educational paths, México.

\section{Cómo citar/Citation}

Ramírez López, Mónica (2019). El ingreso a carreras de alta o baja demanda en una universidad mexicana: ¿qué influye en la elección?. Revista de Sociología de la Educación-RASE, 12 (2), 191-208. http://dx.doi.org/10.7203/RASE.12.2.14750. 


\section{Introducción}

A partir de los años 70 las universidades mexicanas experimentaron un aumento en el ingreso de estudiantes a sus aulas. Esta masificación respondió a cambios demográficos, una mayor participación de la población en general a los niveles educativos previos, así como a movimientos sociales que permitieron ampliar las oportunidades de ingreso a sectores que antes estuvieron incluidos, ya sea con la creación de nuevas instituciones o de espacios en las ya existentes.

Las crisis económicas experimentadas en la década de los 80 frenaron el aumento de la matrícula universitaria y el desarrollo del sistema de educación superior, para continuar su crecimiento en los años posteriores. Hoy en día el número de estudiantes que ingresa a la educación superior en México registra un crecimiento continuo, la tasa de cobertura a nivel nacional alcanza el 38,4\% (SNIEG, 2019), porcentaje que contrastado con otros países como Estados Unidos o Europa donde el proceso de masificación ocurrió antes que en México, muestra que la universalización de la cobertura aún está en proceso de desarrollo y consolidación.

La ampliación de la cobertura ha conllevado una diversificación de los sectores universitarios distinta al estudiante típico o tradicional (joven, hombre, perteneciente a las clases altas, de tiempo completo) que imperaba en las universidades mexicanas. Sin embargo, debido a las dificultades de crecimiento de las Instituciones de Educación Superior (IES) públicas en México y a las limitaciones en los recursos públicos destinados a este sector, hoy en día la demanda supera la oferta en las principales IES mexicanas, dando lugar a un sistema de educación superior altamente selectivo (Guzmán y Saucedo, 2010).

Esta situación pone en la mesa diversos temas, entre los que destaca, la construcción de nuevas formas de desigualdad educativas en el acceso, la permanencia y rendimiento de los estudiantes universitarios. De ahí que conocer quiénes son estos estudiantes y los recursos (económicos, materiales, de conocimientos y habilidades) con los que cuentan para desarrollar sus estudios universitarios resulta de vital para el diseño de estrategias, acciones y políticas, institucionales y públicas, que favorezcan sus trayectorias y egreso oportuno (De Garay, 2004).

Las investigaciones en el campo educativo resaltan que ligado a estas desigualdades se encuentra la influencia de distintos factores. Entre ellos, las características socioeconómicas, educativas y culturales de los núcleos familiares (Jackson, 2013; Davey, 2012; Bartolucci, 2017; Guzmán y Serrano, 2010), así como resultados académicos de los niveles educativos previos por medio del promedio, tiempo en concluir los estudios previos, etc. (De Garay et al., 2016; Chain, 2003; Bartolucci, 1994), como a través de la autopercepción que tiene los estudiantes de sus capacidades (Torrents, 2016).

Estos factores inciden en las elecciones que realizan los estudiantes, como el continuar sus estudios universitarios, las instituciones a las que asistirán y la carrera que estudiarán. Respecto a esta última elección, se ha comprobado que elegir carreras de alta o baja demanda, las cuales presentan un grado diferente de selección, tiene efectos en las trayectorias universitarias de los estudiantes (Romo y Fresán, 2001) y que puede ser el resultado de una estrategia para garantizar un lugar en un sistema universitario, a pesar de no ser la opción deseada (Guzmán y Serrano, 2010).

El objetivo del trabajo es explorar, a través del análisis de datos sobre una muestra de estudiantes de la Universidad Nacional Autónoma de México (UNAM), la relación entre las condiciones sociales y culturales de la familia de los estudiantes y las características académicas que presentan al momento de inscribirse sobre las probabilidades de elección de una carrea de alta o baja demanda. 
El resto del artículo se organiza de la siguiente manera. En primer lugar, se hace una revisión de los estudios respecto al acceso a la educación superior desde la sociología, así como un esbozo de la situación en México y del ingreso a la UNAM. Seguidamente, se describen los datos que se utilizan en la parte empírica, así como la conformación de variables y del modelo logístico realizado. Posteriormente, se ofrece una caracterización de los estudiantes de la muestra y los resultados respecto a los factores que inciden en la elección de carreras de alta y baja demanda. Finalmente, el artículo cierra con algunas reflexiones generales.

\section{El ingreso a la educación superior: la investigación al respecto}

A partir de los procesos de masificación que han vivido las universidades a lo largo del mundo se enfatiza la necesidad de conocer quiénes son los estudiantes que acceden a la educación superior: sus características y condiciones y cómo estas tienen alguna incidencia o no en el desarrollo de sus estudios.

Una importante línea de investigación en la sociología de la educación se ha dedicado al análisis del logro académico de los estudiantes en los distintos niveles educativos. Desde los años 60 algunas investigaciones mostraban la importancia de las características familiares en los aprendizajes de los estudiantes (Coleman, 1966). A partir de ellos, el debate ha continuado y se han sumado corrientes que enfatizan el rol de los factores culturales en el desempeño educativo (Bourdieu y Passeron, 1977).

Diversas investigaciones en educación concuerdan en mencionar que el nivel y logros educativos están en gran parte afectados por el origen social, en particular la clase social y los niveles de educación alcanzados por los padres (Van Werfhorst et al., 2005; Goldthorpe, 1996) ya que promueven y transmiten habilidades cognitivas, valores, actitudes, creencias, predisposiciones y comportamientos, a la par de contar con el sustento económico para apoyar la formación de los hijos.

En la investigación educativa en México, el concepto de capital cultural se ha adaptado para el análisis del rendimiento en diversos niveles educativos al considerar tanto los recursos educativos (Casillas et al., 2007; De Garay, 2004), como bienes materiales en el hogar de los estudiantes. Desde esta perspectiva, la herencia familiar asegura el sustento económico y una serie de recursos culturales, simbólicos, redes y los códigos dominantes de la cultura escolar que posibilitan el acceso a las mejores oportunidades educativas.

Por otro lado, una veta de trabajos menciona cómo el origen social tiene una presencia preponderante en los procesos de elección que se presentan en las distintas bifurcaciones del sistema educativo (Mare, 1981), tanto en la continuación de los estudios, como respecto a las opciones tanto institucionales como de carreras que se consideran como plausibles y viables (Ball et al., 2002; Davey, 2012).

Desde esta perspectiva el rendimiento en los estudios previos al nivel superior influye preponderantemente en las elecciones educativas y en el logro universitario (Beaupère y Boudesseul, 2009). Los resultados de la trayectoria académica de los estudiantes pueden ser entendidos como los efectos primarios (Boudon, 1974; Breen y Goldthorpe, 1997) que, combinados con las características de origen social (efectos secundarios) tienen una influencia al definir probabilidades de éxito y elecciones educativas (Shavit et al., 2007).

Los efectos primarios y secundarios pueden dar lugar a efectos de compensación, es decir, al ajuste de planes y elecciones dependiendo los resultados académicos de los niveles previos diferenciados por clase social; o de información incompleta, que se da por la interpretación diferenciada que las familias y los estudiantes hacen sobre los resultados académicos (Bernardi y Cebolla, 2014). 
Los orígenes sociales no actúan en solitario, interactúan con atributos sociodemográficos como el sexo, la edad o la pertenencia a grupos étnicos influyendo en el acceso, la elección de carreras, la condición estudiantil, el rendimiento y conclusión de los estudios (Bartolucci, 1994, 2017; Buquet et al., 2013).

Aunado a estos, se encuentra también la combinación de los efectos del origen social con las diferencias en los sistemas educativos. Esta se plasma en la asignación de los estudiantes a opciones educativas estratificadas a lo largo de su trayecto escolar (Lucas, 2001), lo cual tendrá incidencia en sus resultados académicos. Esta línea de trabajos demuestra cierto efecto del entorno institucional, pues «más que una adquisición individual, el logro educativo es un proceso institucionalizado» (Elman y O’Rand, 2007: 1278), de modo que las instituciones previas en las que se estudia tienen un efecto en el ingreso a la educación superior.

Los efectos de tipo institucional están presentes a lo largo del trayecto escolar y para el ingreso al nivel superior, como a nivel bachillerato, influyen también los procesos de ingreso de las instituciones (Hernández, 2015; Acosta, 2013) y el grado de demanda que estas presenten (Rodríguez, 2016).

En este sentido, resulta interesante analizar cómo a pesar de la ampliación de cobertura que se ha dado en los sistemas universitarios, incluido el mexicano, continúan operan los mecanismos de desigualdad económica, cultural y educativa en las elecciones educativas de los estudiantes, en este caso en particular, por carreras de alta y baja demanda.

A pesar de que el sistema de educación superior mexicano ha crecido tanto en matrícula, número de establecimientos, tipo de instituciones y oferta educativa en las últimas décadas (Miller, 2015), no se ha logrado atender la demanda de ingreso de un número significativo de aspirantes que se vuelca sobre unas cuantas carreras universitarias.

Esta situación es producto del incremento de estudiantes que egresa del bachillerato, a la par que las IES públicas no cuentan con los recursos (económicos, humanos y de infraestructura) para dar cabida a esos jóvenes. Una de estas instituciones es la UNAM, una de las principales universidades públicas en México.

El ingreso a la licenciatura a la UNAM se da mediante opciones: por pase reglamentado o concurso de selección. La primera opción opera para aquellos estudiantes que concluyeron el bachillerato en la propia UNAM, ya sea en la Escuela Nacional Preparatoria (ENP) o en el Colegio de Ciencias y Humanidades $(\mathrm{CCH})$, con un promedio mínimo de siete y en un máximo de cuatros años; dependiendo estos resultados los estudiantes tienen derecho a elegir carrera de su preferencia y el plantel en el cual cursarán sus estudios. La segunda opción opera para aquellos estudiantes que provienen de cualquier bachillerato, público o privado, y que cuenten con un promedio mínimo de siete en el nivel previo. En ese caso, aunque los aspirantes eligen la carrera a cursar y el plantel, estos se asignan dependiendo el puntaje mínimo establecido por cada carrera y el cupo que establezca la institución ${ }^{2}$.

Cada año se presenta e incrementa la demanda de ingreso vía el concurso de selección; entre 2001 y 2009 esta aumentó en 88 \%. Aunque la institución aumenta también el número de estudiantes aceptados, solo puede dar cabida a un $8 \%$ del total aspirantes.

Muchos de los jóvenes excluidos intentan en diversas ocasiones conseguir su ingreso, preparándose para el examen, cambiando su elección de carreras o planteles; otros buscan distintas opciones, mientras

2 Los puntajes varían entre las carreras y dependen de los lugares que cada carrera y plantel tiene disponibles, así como la demanda. 
otros más quedan fuera del sistema educativo. Lo cierto es que en este proceso se ponen en juego estrategias, elecciones y recursos desiguales en un sistema altamente selectivo.

\section{Estrategia metodológica: datos, variables y técnicas analíticas}

Los datos que se presentan provienen de un cuestionario llamado Hoja de datos socioeconómicos, el cual, llenan los aspirantes al solicitar ingreso a la UNAM, y cuya responsabilidad está a cargo de la Dirección General de Planeación (DGPL). Este instrumento contiene información sobre la situación socioeconómica y algunos antecedentes académicos de los estudiantes. En conjunto con las historias académicas de los estudiantes, esta información forma parte de un proyecto de investigación en curso que tiene por objetivo el análisis de las trayectorias de estudiantes de licenciatura de la UNAM.

La muestra está conformada por 1378 estudiantes que ingresaron en el ciclo escolar 2013-2014 a ocho carreras con mayor y menor demanda para ese periodo en las cuatro áreas de conocimiento: ciencias físico matemáticas y de las ingenierías, ciencias químico biológicas y de la salud, ciencias sociales y humanidades y artes $^{3}$. Para el artículo se analizaron los datos de los estudiantes de la muestra correspondientes a la situación sociodemográfica, socioeconómica familiar y de su trayectoria escolar previa.

El análisis se divide en dos partes. En un primer momento, se identificaron los tipos de perfiles académicos de la cohorte de estudiantes analizada al momento de ingresar a la UNAM. Para ello se realizó un análisis de clúster que permitió identificar grupos de casos relativamente homogéneos con base en tres variables: el tiempo en que los estudiantes concluyeron los estudios de nivel medio superior, el promedio que obtuvieron en dicho nivel y si presentaron o no exámenes de recuperación o reprobaron alguna materia.

El análisis permitió identificar tres tipos de perfiles académicos de ingreso a la educación superior:

- Ideal: concluyeron el bachillerato en tres años con un promedio alto (8,6 a 10) y sin presentar exámenes de recuperación o recursar materias ( $34 \%$ de los casos).

- Bueno: egresaron en tres años, pero obtuvieron un promedio regular (7 a 8,5) y presentaron exámenes de recuperación o recursar materias ( $35 \%$ de los casos)

- Aceptable: la mayoría de ellos egresaron del nivel medio superior en más de tres años con un promedio regular $(7$ a 8,5$)$ y presentaron exámenes de recuperación y recursaron materias $(31 \%$ de los casos).

Estos perfiles se utilizan para mostrar la caracterización de los estudiantes al ingreso a la UNAM considerando cuestiones referentes a cuestiones sociodemográficas (seo, edad, estado civil), socioeconómicas y culturales de la familia de origen de los estudiantes. Esta dimensión está conformada por diversas variables:

a) La escolaridad máxima de los padres, la cual se agrupó la escolaridad de ambos padres en una variable con cuatro categorías: educación básica, (ambos padres, o al menos uno, con estudios hasta de secundaria), educación media (ambos padres, o al menos uno, con educación media superior), uno de los padres con educación superior (al menos uno de los padres tuvo contacto con educación superior) y ambos con educación superior (ambos padres con escolaridad superior o posgrado).

3 Las carreras con mayor demanda para la generación 2014 son: ingeniería mecatrónica, enfermería, relaciones internacionales y pedagogía; mientras que las de menor demanda: matemáticas, lengua y literaturas modernas (letras italianas), química y geografía. 
b) La condición de escolaridad del estudiante entre sus hermanos, para aproximarse a una medida de capital cultural del núcleo familiar. Para ello se consideró el número de hermanos y la posición del estudiante entre ellos. Así se conformó una variable que permite conocer si el estudiante es el primero en su familia en llegar a la educación superior o si algunos de sus hermanos han accedido ya a estudios universitarios.

c) El tamaño de familia, si los estudiantes continúan viviendo en el hogar familiar (ambos o alguno de sus padres y hermanos) y sobre quién sostiene sus estudios universitarios.

d) La ocupación de los padres se agrupó en una variable resumen usando una adaptación de la clasificación CASMIN respecto a la jerarquía y prestigio de las ocupaciones (Erikson y Goldthorpe, 1992). Las ocupaciones se agregaron en cuatro categorías: actividades manuales de baja, actividades manuales de alta calificación, actividades no manuales de baja y actividades no manuales de alta calificación. Esta variable se complementa con aquella que da cuenta de si el estudiante laboraba al ingresar a la universidad.

En un segundo momento, se analiza la probabilidad de elección de una carera mediante un modelo de regresión logística binomial (Long y Freese, 2006); en el cual, la variable dependiente indica si el estudiante eligió una carrera de baja demanda (0) o una de alta demanda (1); mientras que las variables independientes dan cuenta de: un proxi de origen social de los estudiantes a través del nivel máximo de escolaridad de los padres y su ocupación; la trayectoria previa del estudiante con la inclusión del perfil académico de ingreso de los estudiantes construido a través del análisis de conglomerados, así como una variable que muestra el tipo de bachillerato de procedencia para considerar efectos institucionales; se incluye, además, dos variables sociodemográficas que en diversas investigaciones se ha demostrado tienen una influencia en la elección de carreras: el sexo y la edad de ingreso a la universidad y, finalmente, se agrega el área de conocimiento a la cual pertenecen las carreras. La tabla I muestra la descripción y codificación de estas variables.

Tabla I. Variables para el análisis de la elección del tipo de carrera

\begin{tabular}{|c|c|c|}
\hline VARIABLE & DESCRIPCIÓN & CATEGORIAS \\
\hline \multicolumn{3}{|l|}{ Variable dependiente } \\
\hline \multirow[t]{2}{*}{ Carreras por tipo de demanda } & \multirow{2}{*}{$\begin{array}{l}\text { Variable dicotómica que agrupa las carreras por tipo de } \\
\text { demanda }\end{array}$} & $0=$ Carreras de baja demanda \\
\hline & & $1=$ Carreras con alta demanda \\
\hline \multicolumn{3}{|l|}{ Variables independientes } \\
\hline \multirow[t]{2}{*}{ Sexo } & \multirow[t]{2}{*}{ Sexo del estudiante } & $0=$ Hombres \\
\hline & & $1=$ Mujeres \\
\hline Edad & Edad del estudiante & (variable continua) \\
\hline \multirow[t]{3}{*}{ Índice de escolaridad de los padres } & \multirow[t]{3}{*}{ Escolaridad máxima alcanzada por ambos padres } & 0 = Hasta educación básica \\
\hline & & 1 = Educación media superior \\
\hline & & 2 = Educación superior \\
\hline \multirow[t]{4}{*}{ Índice de ocupación de padres } & \multirow[t]{4}{*}{ Tipo de ocupación de ambos padres } & $0=$ Actividades manuales de baja calificación (MBC) \\
\hline & & 1 = Actividades manuales de alta calificación (MAC) \\
\hline & & 2 = Actividades no manuales de baja calificación (NMBC) \\
\hline & & $3=$ Actividades no manuales de alta calificación (NMAC) \\
\hline
\end{tabular}




\begin{tabular}{|c|c|c|}
\hline Perfil académico de ingreso & $\begin{array}{l}\text { Resultados de los estudiantes de la trayectoria escolar } \\
\text { previa }\end{array}$ & $\begin{array}{l}0=\text { Aceptable } \\
1=\text { Bueno } \\
2=\text { Ideal }\end{array}$ \\
\hline Escuela de procedencia & Tipo de bachillerato en que el estudiante curso el nivel & $\begin{array}{l}0=0 \text { tras } \\
1=\text { Escuela Nacional Preparatoria } \\
2=\text { Colegio de Ciencias y Humanidades }\end{array}$ \\
\hline Área de conocimiento de la carrera & $\begin{array}{l}\text { Campo o área de conocimiento de la carrera a la que está } \\
\text { inscrita el estudiante }\end{array}$ & $\begin{array}{l}0=\text { Cs. Físico matemáticas y de las ingenierías } \\
1=\text { Cs. químico biológicas y de la salud } \\
2=\text { Cs. sociales } \\
3=\text { Humanidades y artes }\end{array}$ \\
\hline
\end{tabular}

Fuente: elaboración propia.

4. Perfiles académicos de ingreso y condiciones socio demográficas, económicas y culturales de los estudiantes de la UNAM

La caracterización que se presenta a continuación toma como punto de contraste los perfiles académicos con que ingresan los estudiantes a la universidad y se divide en tres dimensiones: cuestiones sociodemográficas, cuestiones económicas y culturales de las familias de los estudiantes, y aspectos de su ingreso a la UNAM que, como se menciona en diversas investigaciones, influyen en el ingreso a la educación superior.

Las principales características sociodemográficas por perfiles académicos de ingreso de los estudiantes se resumen en la tabla II.

Tabla II. Estadísticas descriptivas sociodemográficas por tipo de perfil académico de ingreso de los estudiantes (porcentajes)

\begin{tabular}{|c|c|c|c|c|}
\hline \multicolumn{5}{|c|}{ TIPO DE PERFIL ACADÉMICO } \\
\hline & $\begin{array}{l}\text { ACEPTABLE } \\
\text { (424 CASOS) }\end{array}$ & $\begin{array}{c}\text { BUENO } \\
\text { (479 CASOS) }\end{array}$ & $\begin{array}{c}\text { IDEAL } \\
\text { (467 CASOS) }\end{array}$ & $\begin{array}{l}\% \text { TOTAL } \\
(\mathrm{N}=1370)\end{array}$ \\
\hline \multicolumn{5}{|l|}{ Sexo } \\
\hline Hombre & 49,1 & 45,9 & 45,2 & 46,6 \\
\hline Mujer & 50,9 & 54,1 & 54,8 & 53,4 \\
\hline \multicolumn{5}{|l|}{ Edad } \\
\hline Hasta 19 & 38,7 & 83,7 & 90,6 & 72,1 \\
\hline De 20 a 24 & 48,6 & 14 & 9,2 & 23,1 \\
\hline De 25 en adelante & 12,7 & 2,3 & 0,2 & 4,8 \\
\hline \multicolumn{5}{|l|}{ Estado civil } \\
\hline Soltero & 91,5 & 99,2 & 99,6 & 96,9 \\
\hline Otros & 8,5 & 0,8 & 0,4 & 3,1 \\
\hline \multicolumn{5}{|l|}{ Tiene hijos } \\
\hline No & 89,9 & 98,8 & 100 & 96,4 \\
\hline Sí & 10,1 & 1,2 & 0 & 3,6 \\
\hline
\end{tabular}

Fuente: elaboración propia. 
De manera general, entre los estudiantes que conforman la muestra predominan las mujeres: 53,2\% en relación con $47 \%$ de los hombres. Como los datos lo manifiesta a nivel mundial la presencia de las mujeres en las universidades se ha incrementado de forma progresiva: entre 2000 y 2017 el porcentaje de mujeres inscritas en educación superior pasó de 18,9\% a 40,1\% (Banco mundial, 2019).

En México los datos corresponden a la tendencia mundial: las mujeres representan el 50,3\% de la matrícula nacional en el ciclo 2017-2018. Aunque, como se verá más adelante, su participación en algunos campos de conocimiento continúa siendo minoritaria. Si se observa el porcentaje de mujeres por perfil académico se tiene una mayor presencia de estas en los tres perfiles, con una diferencia a favor de 4 puntos porcentuales en los perfiles bueno e ideal.

Respecto a la edad, la mayoría de ellos $(72 \%)$ se encuentran en el rango etario normativo de ingreso a la educación superior; es decir, tienen hasta 19 años. Un porcentaje menor agrupa a estudiantes entre los 20 a 24 años (23\%) y a quienes tienen más de 25 años (5\%) al ingresar a la UNAM. Las diferencias por perfil son importantes respecto a la edad: el perfil ideal agrupa en su mayoría (90,6\%) a estudiantes jóvenes; mientras que este porcentaje baja considerablemente entre los estudiantes que conforman el perfil aceptable $(38,7 \%)$.

Este último dato podría dar cuenta de dos situaciones problemáticas en el sistema educativo mexicano: a) de cierto desfase o rezago de algunos de estos estudiantes en algún nivel educativo previo, o bien, b) del alargamiento entre el egreso del nivel bachillerato y la entrada a la educación superior como consecuencia de diversos intentos por ingresar a una carrera universitaria.

Al tratarse de una población principalmente joven, la gran mayoría de ellos son solteros (97\%) y sin hijos (96\%). Aunque al analizarlo por tipo de perfil se observa que, para el perfil aceptable, 1 de cada 10 manifiestan ser casados o vivir en unión libre y contar con hijos. Estos datos no son menores, ya otras investigaciones han marcado la diferencia entre los jóvenes que se encuentran realizando estudios universitarios y aquellos que han salido del sistema educativo, al asumir responsabilidades propias del paso a la vida adulta: la salida del hogar parental, el ingreso al primer empleo, la primera unión y el nacimiento del primer hijo.

¿Cuáles son las características socioeconómicas y culturales de las familias de los estudiantes? Como se observa en la tabla III, en general, la mayoría de los estudiantes continúa viviendo con sus padres y/o hermanos (92,3\%), aunque este porcentaje es menor para el tipo de perfil aceptable (87,2\%). Más de la mitad de los estudiantes $(56 \%$ ) proviene de familias pequeñas (uno a dos hijos), manteniéndose este porcentaje entre los perfiles de los estudiantes, y un 13,7\% de ellos son hijos únicos en su familia.

Tabla III. Estadísticas descriptivas socioeconómicas y culturales por tipo de perfil académico de ingreso de los estudiantes (porcentajes)

\begin{tabular}{|c|c|c|c|c|}
\hline \multicolumn{5}{|c|}{ TIPO DE PERFIL ACADÉMICO } \\
\hline & $\begin{array}{l}\text { ACEPTABLE } \\
\text { (424 CASOS) }\end{array}$ & $\begin{array}{c}\text { BUENO } \\
\text { (479 CASOS) }\end{array}$ & $\begin{array}{c}\text { IDEAL } \\
\text { (467 CASOS) }\end{array}$ & $\begin{array}{l}\text { \% TOTAL } \\
(\mathrm{N}=1370)\end{array}$ \\
\hline \multicolumn{5}{|l|}{ Con quién vives } \\
\hline Padres, hermanos & 87,2 & 93,6 & 95,9 & 92,3 \\
\hline \multicolumn{5}{|l|}{ Tipo de familia } \\
\hline Pequeña & 51,7 & 55,5 & 61,9 & 56,5 \\
\hline Mediana & 41,8 & 40,7 & 35,1 & 39,1 \\
\hline
\end{tabular}




\begin{tabular}{|c|c|c|c|c|}
\hline Grande & 6,6 & 3,8 & 3,0 & 4,4 \\
\hline \multicolumn{5}{|c|}{ Persona que sostiene estudios } \\
\hline Ambos padres & 33,1 & 42,5 & 34,9 & 36,9 \\
\hline Padre & 28,7 & 33,5 & 43,6 & 35,3 \\
\hline Madre & 18,4 & 16,2 & 18,4 & 17,6 \\
\hline Él mismo & 15,0 & 6,4 & 1,2 & 7,3 \\
\hline Otros & 4,8 & 1,5 & 2,1 & 2,8 \\
\hline \multicolumn{5}{|l|}{ Trabaja } \\
\hline No & 51,7 & 73,0 & 81,6 & 69,1 \\
\hline Sí & 48,3 & 27,0 & 18,4 & 30,9 \\
\hline \multicolumn{5}{|l|}{ Ocupación de los padres } \\
\hline Manual baja calificación & 8,9 & 9,4 & 7,63 & 8,6 \\
\hline Manual alta calificación & 25,0 & 22,1 & 19,7 & 22,2 \\
\hline No manual baja calificación & 53,9 & 53,7 & 53,7 & 53,8 \\
\hline No manual alta calfificación & 12,3 & 14,9 & 19,0 & 15,5 \\
\hline \multicolumn{5}{|l|}{ Escolaridad de los padres } \\
\hline Básica & 23,4 & 22,2 & 19,1 & 21,5 \\
\hline Media superior & 33,9 & 33,6 & 28,7 & 32,0 \\
\hline $\begin{array}{l}\text { Al menos uno con educcación } \\
\text { superior }\end{array}$ & 27,7 & 23,5 & 23,1 & 24,6 \\
\hline \multicolumn{5}{|l|}{ Escolaridad entre hermanos } \\
\hline $\begin{array}{l}\text { Primero en acceder a educación } \\
\text { superior }\end{array}$ & 57,3 & 54,3 & 58,7 & 56,7 \\
\hline $\begin{array}{l}\text { Otros hermanos con educación } \\
\text { superior }\end{array}$ & 31,6 & 31,3 & 26,1 & 29,6 \\
\hline Hijo único & 11,1 & 14,4 & 15,2 & 13,7 \\
\hline
\end{tabular}

Fuente: elaboración propia.

$\mathrm{Al}$ indagar sobre las condiciones materiales y económicas con las que cuentan los estudiantes en sus hogares se observa de manera general, que son principalmente ambos padres quienes sostienen económicamente a los estudiantes (37\%), seguido únicamente de padre $(35,5 \%)$ y la madre $(17,6 \%)$ de manera individual. Estos últimos datos podrían sugerir núcleos familiares donde las madres no tendrían la necesidad de trabajar, o bien, núcleos familiares donde las madres representan el único o principal sostén familiar.

$\mathrm{Al}$ analizar por tipo de perfil se observa que entre los estudiantes con perfil ideal es mayor la proporción del padre que sostiene los estudios (43,5\%), mientras que entre los estudiantes del perfil aceptable se incrementa la proporción de estudiantes que sostienen ellos mismo sus estudios (15\%).

Dos situaciones se exploran a partir de estos datos: 1) la condición laboral de los estudiantes y el tiempo que dedicaban a ello a su ingreso a la universidad, y 2) las ocupaciones de los padres de estudiantes, en especial de aquellos que solo cuentan con el sustento de uno de ellos.

$\mathrm{Al}$ analizar si los estudiantes trabajan o no y el tiempo que dedican a ello se observa que, de manera general, la mayoría de los estudiantes $(69 \%)$ no desempeñaban alguna actividad laboral al ingresar a la universidad, y aquellos que laboraban, lo hacían por temporadas (16,2\%), dedicando menos de 16 horas 
a la semana (13\%). Los porcentajes entre quienes trabajan o no cambian al analizarlo por perfiles: para aquellos del perfil ideal el porcentaje de quienes no laboran llega a $81 \%$, para los del perfil bueno disminuye a $73 \%$ y finalmente, para los del perfil aceptable el porcentaje llega a $51 \%$.

Esta situación refleja cómo la dedicación de actividades laborales está compitiendo con el tiempo que los estudiantes pueden dedicar a los estudios, y que incluso puede estar jugando de manera negativa en su rendimiento académico. Como mencionan algunos autores (De Garay et al., 2017; Guzmán, 2004) una importante proporción de estos estudiantes-trabajadores mantiene esta condición durante sus estudios universitarios, lo que repercutirá en su integración y permanencia ya que la universidad no constituirá para estos estudiantes, uno de los espacios principales de referencia (Saraví, 2015).

¿Cuánto apoyo brindan los padres a sus estudiantes? En relación con la cuestión económica no se cuenta con una variable que de cuenta de los ingresos familiares. Una aproximación puede tenerse al analizar las ocupaciones de los padres. Como se muestra en la tabla III la mayoría de los padres de los estudiantes laboran en ocupaciones no manuales de baja calificación (53,8\%), es decir como empleados especializados, docentes no universitarios, dueños de pequeños comercios, etc. Esta proporción se mantiene entre los tres perfiles. De manera general, casi una tercera parte de las madres de los estudiantes (32\%) no labora y una cuarta parte de los padres se desempeña en actividades manuales de baja calificación (técnicos, trabajadores de oficina, realizando algún oficio o por su cuenta).

$\mathrm{Al}$ analizar aquellos casos que reportaron contar con el sustento de uno de los padres, se observa que tanto padres como madres trabajan en actividades no manuales de baja calificación, aunque la proporción difiere, para el caso de quienes señalaron a la madre como principal sostén el porcentaje llega a $60 \%$, mientras que, en el caso del padre, es del 50\%. Otra cuestión por destacar es que cuando se menciona al padre como principal sostén, el porcentaje de madres que no labora es de $73 \%$; en el caso contrario, madres como principal sostén y padres que no laboran, la proporción es mucho menor $23 \%$.

Otro elemento por analizar en la caracterización de la muestra de estudiantes se refiere al contacto en su familia con la educación superior. En palabras de Bourdieu (1977), el capital cultural en su modo institucionalizado que, diversas investigaciones en el plano internacional y nacional han mostrado como el nivel de educación alcanzado por los padres tiene una influencia en la progresión educativa, en el acceso a la educación superior y a diferentes instituciones. Al analizar el nivel máximo de estudios alcanzado por los padres de la muestra de estudiantes se tiene que el nivel educativo con mayor proporción lo representa el bachillerato (32\%), solo $22 \%$ de ambos padres de los estudiantes cuentan con estudios universitarios y en una proporción similar $(21,5 \%)$ de los padres concluyeron hasta el nivel básico de educación.

$\mathrm{Al}$ analizar este indicador por tipo de perfil académico de los estudiantes se observa que es entre los estudiantes de perfil ideal que ambos padres cuentan con estudios universitarios (29\%). Para los perfiles bueno $(34 \%)$ y aceptable (34\%), predomina el que los padres cuenten con el nivel bachillerato como grado máximo de estudios.

El acceso a la educación superior entre los hermanos, descartando aquellos que son hijos únicos (14\%), evidencia que 2 de cada 3 de los estudiantes son los primeros entre sus hermanos en acceder a la educación superior, mientras que un tercio cuenta con hermanos con educación superior. Destaca que entre los estudiantes del perfil ideal el porcentaje de «pioneros» (Bourdieu, 1977) es ligeramente mayor a los otros perfiles (58,7\% vs 57,3\% y 54,2\%), probablemente esto se deba a que los estudiantes analizados sean el mayor o uno de los mayores entre sus hermanos. 
Finalmente, para concluir la caracterización de los estudiantes de la muestra, en la tabla IV se presentan algunos indicadores de la trayectoria escolar previa de los estudiantes de acuerdo con su perfil de ingreso.

\section{Tabla IV. Estadísticas descriptivas sobre aspectos de trayectoria académica previa por tipo de perfil académico de ingreso de los estudiantes (porcentajes)}

\begin{tabular}{|c|c|c|c|c|}
\hline \multicolumn{5}{|c|}{ TIPO DE PERFIL ACADÉMICO } \\
\hline & $\begin{array}{l}\text { ACEPTABLE } \\
\text { (424 CASOS) }\end{array}$ & $\begin{array}{c}\text { BUENO } \\
\text { (479 CASOS) }\end{array}$ & $\begin{array}{c}\text { IDEAL } \\
\text { (467 CASOS) }\end{array}$ & $\begin{array}{l}\begin{array}{c}\% \text { TOTAL } \\
(\mathrm{N}=1370)\end{array}\end{array}$ \\
\hline \multicolumn{5}{|l|}{ Escuela de procedencia } \\
\hline $\begin{array}{l}\text { Escuela Nacional Preparatoria } \\
\text { (ENP) }\end{array}$ & 33,5 & 42,8 & 41,1 & 39,2 \\
\hline $\begin{array}{l}\text { Colegio de Ciencias y Humanidades } \\
\text { (CCH) }\end{array}$ & 35,6 & 37,2 & 28,7 & 33,8 \\
\hline Otras & 30,9 & 20,0 & 30,2 & 26,9 \\
\hline \multicolumn{5}{|l|}{ Tipo de ingreso } \\
\hline Pase reglamentado & 69,1 & 80,0 & 69,8 & 73,1 \\
\hline Concurso de ingreso & 30,9 & 20,0 & 30,2 & 26,9 \\
\hline \multicolumn{5}{|l|}{ Área de conocimiento } \\
\hline $\begin{array}{l}\text { Cs. física matemáticas } \\
\text { y de las ingenierías }\end{array}$ & 20,5 & 18,2 & 41,3 & 26,8 \\
\hline Cs. biológicas, químicas & 39,2 & 33,8 & 12,4 & 28,2 \\
\hline Cs. sociales & 25,9 & 31,3 & 32,3 & 30,0 \\
\hline Humanidades y artes & 14,4 & 16,7 & 13,9 & 15,0 \\
\hline \multicolumn{5}{|l|}{ Percepción de éxito académico } \\
\hline Regular & 29,3 & 10,6 & 1,3 & 13,2 \\
\hline Bueno & 59,7 & 71,4 & 47,1 & 59,5 \\
\hline Excelente & 11,0 & 18,0 & 51,6 & 27,3 \\
\hline
\end{tabular}

Fuente: elaboración propia.

De manera general, la mayoría de los estudiantes analizados $(73,1 \%)$ provienen del sistema de bachillerato de la UNAM, por lo que ingresaron por el sistema de paso reglamentado. Esta proporción cambia ligeramente entre los estudiantes de cada perfil: para los estudiantes del perfil aceptable e ideal 7 de cada 10 estudiantes ingresó por pase reglamentado, mientras que, del perfil bueno, la proporción se incrementa a 8 de cada 10 estudiantes.

No obstante venir del sistema de bachillerato de la UNAM, la proporción varía de acuerdo con el tipo de institución del que se proviene: para aquellos del perfil aceptable la mayoría (35,6\%) egresaron del $\mathrm{CCH}$, por su parte los estudiantes del perfil bueno e ideal egresaron principalmente de la ENP (42,8\% y $41,1 \%$ respectivamente).

En cuanto a las áreas de conocimiento de las carreras en las que ingresan los estudiantes, se tiene una diferencia al observar la distribución por perfiles académicos. Aquellos estudiantes que conforman el perfil aceptable y bueno eligen principalmente carreras del área ciencias químico-biológicas y de la salud (39,2\% y 33,8\% respectivamente) y de las ciencias sociales (25,9\% y $31,3 \%$ respectivamente); mientras que los estudiantes del perfil ideal se inscriben a carreras del área de ciencias físico matemáticas y de las 
ingenierías (41,3\%) seguida de carreras del área de ciencias sociales (32,3\%). Es importante mencionar que, al analizar este dato por sexo se tiene que solo una de cada 10 estudiantes se encuentra inscrita en carreras relacionadas con física, matemáticas o ingeniería en comparación a 4 de cada 10 hombres.

Por último, se exploró también la respuesta de los estudiantes por tipo de perfil respecto a su percepción de éxito académico hasta el momento de su ingreso a la universidad. Destaca que, este guarda cierta correspondencia con los perfiles identificados: aquellos del perfil aceptable y bueno consideran principalmente su éxito académico como bueno (59,7\% y 71,4\% respectivamente), mientras que entre los estudiantes que conforman el perfil ideal, la mayoría considera como excelente su éxito académico $(51,6 \%)$.

\section{Factores asociados a la elección de carreras de alta o baja demanda}

Para conocer los factores asociados a la probabilidad de elegir una carrera universitaria de alta o baja demanda se realizó un modelo de regresión logística binomial que permite analizar las probabilidades asociadas a esta elección controlando simultáneamente variable demográficas, socioeconómicas y culturales y de la trayectoria escolar previa de los estudiantes.

En total se ajustaron 4 modelos en los que se incorporaron sucesivamente variables de las distintas dimensiones analizadas; las razones de momios de cada una de las categorías de las variables incluidas y su significación se muestran en la tabla $\mathrm{V}^{4}$.

Tabla V. Factores asociados a la elección de carreras de alta o baja demanda. Modelos de regresión logística. Razones de momios

\begin{tabular}{lcccc}
\hline \multicolumn{1}{c}{$\begin{array}{c}\text { VARIABLES } \\
\text { INDEPENDIENTES }\end{array}$} & MODELO 1 & MODELO 2 & MODELO 3 & MODELO 4 \\
\hline $\begin{array}{l}\text { Sexo } \\
\text { (referencia: hombres) }\end{array}$ & $2,47^{\star \star \star}$ & $2,45^{\star \star \star}$ & $2,59^{\star \star \star}$ & $1,64^{\star \star \star}$ \\
\hline Edad & 1,04 & 1,03 & $1,13^{\star \star \star}$ & $1,11^{\star \star}$ \\
\hline
\end{tabular}

Escolaridad de los padres

(referencia: educación básica)

\begin{tabular}{lccc}
\hline Media superior & 1,42 & $1,76^{\star \star}$ & $1,62^{\star}$ \\
\hline Superior & $1,55^{\star}$ & $1,86^{\star \star}$ & $1,64^{\star}$
\end{tabular}

Ocupación de los padres

(referencia: manuales baja

calificación)

\begin{tabular}{lccc}
\hline Manuales alta calificación & $0,58^{\star}$ & $0,56^{\star}$ & $0,50^{\star}$ \\
\hline No manuales baja calificación & 0,64 & 0,67 & 0,63 \\
\hline No manuales alta calificación & 0,74 & 0,80 & 0,80 \\
\hline
\end{tabular}

Perfil académico

(referencia: aceptable)

\begin{tabular}{lrr}
\hline Bueno & 1,06 & 0,93 \\
\hline Ideal & $4,77^{\star \star \star}$ & $6,12^{\star \star \star}$ \\
\hline
\end{tabular}

Escuela de procedencia

(referencia: otras)

\begin{tabular}{lll}
\hline ENP & 1,32 & $1,66^{\star \star}$ \\
\hline
\end{tabular}

$\overline{4}$ Se consideraron como medidas de bondad de ajuste de los modelos la pseudo R2, el Bayesian Information Criterion (BIC) y el Akaike Information Criterion (AIC), así como el contraste de la bondad de ajuste entre modelos a través de pruebas de razón de verosimilitud (Long y Freese, 2006). 


\begin{tabular}{|c|c|c|c|c|}
\hline $\begin{array}{c}\text { VARIABLES } \\
\text { INDEPENDIENTES }\end{array}$ & MODELO 1 & MODELO 2 & MODELO 3 & MODELO 4 \\
\hline $\mathrm{CCH}$ & & & $1,95^{\star \star \star}$ & $2,55^{\star \star \star}$ \\
\hline \multicolumn{5}{|c|}{$\begin{array}{l}\text { Área de conocimiento } \\
\text { (referencia: físico matemáticas } \\
\text { y de las ingenierías) }\end{array}$} \\
\hline Cs. biológicas & & & & $1,86^{\star \star}$ \\
\hline Cs. sociales & & & & $2,17^{\star \star \star}$ \\
\hline Humanidades y artes & & & & $20,39^{\star \star \star}$ \\
\hline $\mathrm{N}$ & 1278 & 1278 & 1278 & 1278 \\
\hline Log likelihood & $-774,76106$ & $-768,78457$ & $-708,1315$ & $-645,31113$ \\
\hline Pseudo R2 & 0,0366 & 0,0441 & 0,1195 & 0,1980 \\
\hline AIK & 1555,5221 & 1553,5691 & 1440,263 & 1320,6223 \\
\hline $\mathrm{BIC}$ & 1570,7368 & 1594,1417 & 1501,1219 & 1396,6959 \\
\hline
\end{tabular}

Fuente: elaboración propia.

Niveles de significancia estadística: ${ }^{*} \mathrm{p}<0,05 ;{ }^{* *} \mathrm{p}<0,01 ;{ }^{* * *} \mathrm{p}<0,001$

De manera general la mayoría de las variables y sus categorías son significativas estadísticamente en las probabilidades de elección de una carrera de alta demanda. Para algunas de las categorías de estas variables su magnitud y significación se incrementa o disminuye al introducir variables en los modelos subsecuentes.

Así, en el primero modelo se introdujeron variables sociodemográficas, de las cuales, solo ser mujer resultó estadísticamente significativa y efecto positivo en la elección de carreras con alta demanda (con momios de 2,47 y p <0.001). En el segundo modelo, al introducir variables de corte socioeconómicas y culturales, los momios de ser mujeres descendieron ligeramente, mientras que, el que los padres de los estudiantes cuenten con estudios universitarios tiene una influencia positiva y significativa para elegir carreras de alta demanda; no así el que los padres cuenten con ocupaciones manuales de alta calificación, que reduce los momios estimados de elegir una carrera de alta demanda en 42 por ciento frente a quienes cuentan con una ocupación manual de baja calificación. Estos datos nos indican que existen efectos del origen social asociados a la elección.

$\mathrm{Al}$ incluir en el modelo 3 las variables que conforman la dimensión de trayectoria académica previa, se observa un incremento en los momios de ser mujer y en la edad, esta última además adquiere significatividad estadística, probablemente por la relación que existe con los perfiles académicos. La categoría de escolaridad de los padres aumenta también y gana significatividad respecto a contar con educación básica. De las variables relacionadas con la trayectoria académica, el perfil académico ideal presenta efectos positivos y alta significatividad estadística; al igual que provenir de un $\mathrm{CCH}$ (momios de 1,95 y p <0.001) en contraste con haber realizado los estudios previos en otras instituciones. Esto también corrobora que algunos efectos sociodemográficos y de origen social son medianos por los resultados o «éxito» académico de los estudiantes (Torrenst, 2016) y a un efecto de desigualdad horizontal ligado a la asistencia a diferentes tipos de escuelas en el nivel educativo previo (Solís, 2014).

En el último modelo se introduce el área de conocimiento de la carrera a la que ingresaron los estudiantes. Las categorías de esta variable resultan significativas en distintos niveles con efectos positivos. Su inclusión en el modelo cambia los coeficientes para algunas variables: reduce casi a la mitad los momios de ser mujer (de 2,59 a 1,64), la edad, ambas categorías de la escolaridad de padres y la ocupación de los 
padres. Por otro lado, aumenta los coeficientes del perfil académico ideal y de las categorías de escuela de procedencia, otorgando significatividad a provenir del sistema de la ENP. Es en este último modelo que se analizará el efecto de las diferentes variables incluidas, ya que presenta un mejor ajuste.

El modelo cuatro nos indica que existen efectos tanto sociodemográficos, de origen social y de la trayectoria previa de los estudiantes. Ser mujer incrementa las probabilidades de elegir una carrera de alta demanda en 64 por ciento en comparación con ser hombre. En cuanto a la edad los momios se incrementan en 11 por ciento por cada aumento en años de los estudiantes; como menciona Guzmán (2010) tal vez una mayor edad hable de estudiantes que al no haber sido aceptados en su primer intento se preparen más y adquieran mayor experiencia y madurez a la hora de elegir.

Respecto al origen social familiar, la educación alcanzada por ambos padres incrementa las posibilidades de elegir una carrera de alta demanda en 62 y 64 por ciento para aquellos estudiantes cuyos padres tienen educación a nivel de bachillerato y superior respectivamente. Lo cual indica que a mayor educación de los padres mayores oportunidades de elección de carreras altamente demandadas. No así para la ocupación de los padres, en la que únicamente resultó significativa estadísticamente y con un efecto negativo la categoría que agrupa a padres con ocupaciones manuales de alta calificación, esta reduce en 50 por ciento los chances de los estudiantes al elegir una carrera de alta demanda.

Por otro lado, las variables que dan cuenta de la trayectoria académica previa del estudiante, los momios de elección de carreras de alta demanda se incrementan multiplicativamente por 6 para quienes cuentan con un perfil ideal (término del bachillerato en 3 años, con promedios altos y sin reprobar materias) frente a quienes presentan un perfil aceptable. Por otro lado, egresar del sistema de bachillerato de la UNAM también tiene un efecto positivo en la elección de carreras de alta demanda, aunque no el mismo si se cursó el nivel previo en la ENP o en el CCH; en el primer caso, los momios se incrementan un 66 por ciento respecto a quienes egresaron de otro tipo de escuelas; mientras que para quienes estudiaron en un $\mathrm{CCH}$ la probabilidad aumenta hasta 2,55 veces respecto a haber egresado de otras instituciones. Esto probablemente se deba, como menciona Guzmán (2010) a la cercanía del currículo de estas instituciones que ligado a los antecedentes académicos aseguren el ingreso a carreras de alta demanda, en donde estudiantes provenientes de otras instituciones tienen menores probabilidades de inscribirse, aún cuando presenten trayectorias previas sobresalientes.

En el caso de las variables relativas al área de conocimiento de las carreras en las que se inscriben los estudiantes, todas las categorías son estadísticamente significativas y positivos respecto a la elección de una carrera de alta demanda: los momios de elegir una carrera de alta demanda se incrementan en $86 \%$ para quienes están inscritos en carreas del área de cs. biológicas respecto al área de físico matemáticas; en $117 \%$ para quienes se inscriben a una carrea de cs. sociales y hasta 20,39 por ciento en el área de humanidades y artes en comparación con elegir una carrera del área físico matemáticas y de las ingenierías.

\section{Consideraciones finales}

El objetivo del artículo consistió en caracterizar a los estudiantes que ingresan a una de las principales universidades públicas mexicanas y analizar la incidencia del origen social y los antecedentes académicos de los estudiantes en la probabilidad de elección de carreras de alta y baja demanda.

Respecto a la caracterización de los estudiantes a su ingreso, es posible distinguir diferencias importantes de acuerdo con los perfiles académicos establecidos. Por un lado, destaca la heterogeneidad en las 
características de los estudiantes. Por otro, se observa que, aquellos estudiantes que cuentan con promedios superiores en el bachillerato y que lo concluyeron en tres años son estudiantes principalmente jóvenes, sin obligaciones familiares o laborales, situación que posiblemente contribuyó a contar con una trayectoria continua y con buenos resultados académicos. Estos estudiantes provienen principalmente del bachillerato de la UNAM, en específico, de la Escuela Nacional Preparatoria y están inscritos en carreras del área físico matemáticas y de las ingenierías (ingeniería mecatrónica o matemáticas).

Mientras que, los estudiantes del perfil aceptable, quienes concluyeron su formación de bachillerato en más tiempo del reglamentado, con promedios entre 7-8,5 y que reprobaron o recursaron alguna materia, son estudiantes con edades mayores a las normativas para ingresar a la educación superior, cuentan con responsabilidades familiares y laborales, en contraparte, tienen menores apoyos económicos y también culturales por parte de sus padres. En su mayoría provienen del sistema de bachillerato de la UNAM, principalmente del Colegio de Ciencias y Humanidades. A diferencia de los estudiantes con perfil ideal, estos estudiantes han seleccionado carreras del área de ciencias químico-biológicas y de la salud (enfermería o química).

Respecto a los factores que inciden en las probabilidades de elegir una carrera de alta demanda, se tiene que hay una influencia de diversas dimensiones: sociodemográficas, como el sexo y la edad de los estudiantes; socioeconómicas y culturales en relación con la escolaridad de los padres y su ocupación; de la trayectoria previa sobre los resultados académicos y la institución en que se realizó el nivel de bachillerato, así como el área de conocimiento de la carrera que eligieron.

Es importante mencionar que los cambios al introducir nuevas variables a los modelos anidados sugieren la interacción de estos factores, su exploración queda pendiente en este análisis.

Los factores con mayores momios y con alta significatividad estadística son aquellos que dan cuenta de la trayectoria escolar previa, a través de los resultados plasmados en el perfil académico y de la institución de procedencia, y los relacionados con el área de conocimiento de la carrera elegida.

Estos resultados sugieren una importante participación de los efectos institucionales en las elecciones de los estudiantes. No debe perderse de vista que, de acuerdo con la caracterización realizada por perfiles, la elección por una carrera de alta demanda está sesgada por cuestiones socioeconómicas y demográficas.

Varias preguntas surgen al respecto: ¿cómo toman los estudiantes la elección por las carreras a las que se inscribirán? ¿Con qué información disponen para ello? ¿Qué factores consideran y preponderan al momento de elegir carrera? ¿Esta elección es producto de una estrategia para asegurarse un lugar en la universidad? Estas cuestiones tendrían que profundizarse con estrategias analíticas que permitan conocer y profundizar en los motivos y procesos de decisión de los estudiantes.

Por otro lado, surge la interrogante sobre cómo esta elección tiene incidencia en la permanencia, rendimiento académico y egreso de estos estudiantes de la universidad.

\section{Referencias bibliográficas}

Acosta, Adrián (2013): "Política, actores y decisiones en las universidades públicas en México: un enfoque institucional". Revista de la Educación Superior, 42 (165), 83-100. http://www.scielo.org.mx/scielo. php?script $=$ sci_arttext\&pid=S0185-27602013000100005\&lng $=$ es\&tlng $=$ es. 
Ball, Stephen J.; Davies, Jackie; David, Miriam y Reay, Diane (2002): “Classification and Judgment: Social Class and the Cognitive Structures of Choice of Higher Education". British Journal of Sociology of Education, 23 (1), 51-72. https://doi.org/10.1080/01425690120102854.

Banco Mundial (2019). Indicadores: Inscripción escolar, nivel terciario, mujeres (\% bruto). https://datos. bancomundial.org/indicador/SE.TER.ENRR.FE.

Bartolucci, Jorge (1994). Desigualdad social, educación superior y sociología en México. México: Centro de Estudios Sobre la Universidad México-UNAM/Editorial Porrúa.

Bartolucci, Jorge (2017): "El perfil del alumno de primer ingreso a las licenciaturas de la UNAM: generación 2016-1". Seminario trayectorias y transiciones educativas en México, septiembre 2017, IISUE-UNAM

Beupère, Nathalie y Boudesseul, Gérad (dir.) (2010). Sortir sans diplôme de l’Université. Comprendre les parcours d'étudiants «décrocheurs». Paris: La Documentation Française.

Boudon, Raymond. (1974). Education, Opportunity, and Social Inequality; Changing Prospects in Western Society. New York: Wiley.

Bourdieu, Pierre y Passeron, Jean Claude [1964] (1977). Los herederos. Los estudiantes y la cultura. Buenos Aires: Siglo XXI Editores.

Breen, Richard y Goldthorpe, John H. (1997): "Explaining Educational Differentials: Towards a Formal Rational Action Theory". Rational and Society, 9, 275-305. https://doi.org/10.1177/10434639700900 3002 .

Buquet, Ana; Cooper, Jennifer A.; Mingo Araceli y Moreno Hortensia (2013). Intrusas en la Universidad. México: UNAM-Programa Universitario de Estudios de Género/IISUE.

Casillas, Miguel; Chaín Revuelta, Ragueb y Jácome Nancy (2007): “Origen social de los estudiantes y trayectorias estudiantiles en la Universidad Veracruzana”. Revista de la Educación Superior, XXXVI (2), 7-29. http://publicaciones.anuies.mx/pdfs/revista/Revista142_S1A1ES.pdf.

Chaín Revuelta, Ragueb (2003): “Trayectorias escolares en la Universidad Veracruzana”. Colección pedagógica universitaria, (25-26), 195-235.

Coleman, James (1966). Equality of Educational Opportunity. Washington: Department of Health, Education, and Welfare.

Cuevas de la Garza, José Fernando y de Ibarrola María (2012): "Vidas cruzadas. Los estudiantes que trabajan: un análisis de sus aprendizajes". Revista de la educación superior, 42 (165).

Davey, Gayna (2012): “Beyond a Binary Model of Students’ Educational Decision-Making”. Sociological Research Online, 17 (3) 4. http://www.socresonline.org.uk/17/3/4.html.

De Garay, Adrián (2001). Los actores desconocidos. Una aproximación al conocimiento de los estudiantes. México: Asociación Nacional de Universidades e Instituciones de Educación Superior.

De Garay, Adrián (2004). Integración de los jóvenes en el sistema universitario. Prácticas sociales, académicas y de consumo cultural. México-Barcelona: Pomares.

De Garay, Adrián; Miller, Dinorah y Montoya, Iván (2016): “Una misma institución, estudiantes diferentes. Los universitarios de nuevo ingreso de las unidades Azcapotzalco y Cuajimalpa de la UAM”. So- 
ciológica, 31 (88), 95-140. http://www.sociologicamexico.azc.uam.mx/index.php/Sociologica/article/ view/1145/1133.

Elman, Cheryl y O'Rand, Angela (2007): “The effects of social origins, life events, and institutional sorting on adults' school transitions". Social Science Research, 36, 1276-1299. https://doi.org/10.1016/j. ssresearch.2006.11.001.

Erikson, Robert y Goldthorpe, John (1992). The constant flux: A study of class mobility in industrial societies. Oxford: Clarendon Press.

Goldthorpe, John H. (1996): "Class Analysis and the Reorientation of Class Theory: The Case of Persisting Differentials in Educational Attainment”. British Journal of Sociology, 47, 481-505. https://doi. org/10.2307/591365.

Guzmán Gómez, Carlota (2004). Entre el estudio y el trabajo. La situación y las búsquedas de los estudiantes de la UNAM que trabajan. México: Universidad Nacional Autónoma de México.

Guzmán Gómez, Carlota y Serrano, Olga Victoria (2010): “Las puertas del ingreso a la educación superior: el caso del concurso de selección a la licenciatura de la UNAM”. Revista de la Educación Superior, XL(I), 157, 31-53. https://www.redalyc.org/articulo.oa?id=60420223002.

Guzmán Gómez, Carlota (2005): “Características socioeconómicas, familiares y académicas de los alumnos” en Carlota Guzmán Gómez y Claudia Saucedo Ramos (coords.): La investigación sobre alumnos en México: recuento de una década (1992-2002). México: Consejo Mexicano de Investigación Educativa A.C.

Hernández Fernández, Jimena (2015). Transition to Upper Secondary School in Mexico: New Insights into Selection and Education Expectations. Thesis $\mathrm{PhD}$ in Education. University of Sussex.

Jackson, Michelle (ed.). (2013). Determined to Succeed? Performance Versus Choice in Educational Attainment. Stanford: Stanford University Press.

Long, John Scott y Freese, Jeremy (2006). Regression Models for Categorical Dependent Variables Using Stata. College Station: Stata Press.

Lucas, Samuel R. (2001): "Effectively Maintained Inequality: Education Transitions, Track Mobility, and Social Background Effects". American Journal of Sociology, 106 (6), 1642-1690. https://doi. org/10.1086/321300.

Miller, Dinorah (2015): "Exploración del vínculo entre trayectorias escolares y marcos normativos en la Universidad Autónoma Metropolitana". Revista Iberoamericana de Educación Superior (RIES), México: UNAM-IISUE/Universia, vol. VI (17),2 1-37. https://doi.org/10.1016/j.rides.2015.10.001.

Rodríguez, Eduardo (2016): "El rol de la escuela en las decisiones educativas de sus alumnos bajo el contexto de la transición a las instituciones públicas de educación media superior de la Ciudad de México". Estudios Sociológicos, XXXIV (102), 639-664. http://www.scielo.org.mx/scielo.php?script=sci_ arttext\&pid=S2448-64422016000300639\&lng=es\&tlng=es.

Romo, Alejandra; Fresán Magdalena (2001): "Los factores curriculares y académicos relacionados con el abandono y el rezago" en Ragueb Chaín Revuelta y otros (comps.): Deserción, rezago y eficiencia terminal en las IES. Propuesta metodológica para su estudio. México: ANUIES. 
Saraví, Gonzalo (2015). Juventudes fragmentadas. Socialización, clase y cultura en la construcción de la desigualdad. México: FLACSO-CIESAS.

Shavit, Yossi; Yaish, Meir y Bar-Haim, Eyal (2007): “The Persistence of Persistent Inequality" en Stefani Scherer, Reinhard Pollak, Gunnar Otte y Markus Gangl (eds.): From Origin to Destination. Trends and Mechanisms in Social Stratification Research. Frankfurt y Nueva York: Campus.

SNIEG (Sistema Nacional de Información Estadística y Geográfica) (2019). Catálogo Nacional de Indicadores. https: / www.snieg.mx/cni/escenario.aspx?idOrden $=1.1 \& i n d=6200027780 \&$ gen $=842 \& \mathrm{~d}=\mathrm{n}$.

Solís, Patricio (2014): "Desigualdad social y efectos institucionales en las transiciones educativas" en Emilio Blanco, Patricio Solís y Héctor Robles (coord.): Caminos desiguales. Trayectorias educativas y laborales de los jóvenes en la Ciudad de México. México: El Colegio de México-INEE.

Torrents, Dani (2016): "La autopercepción de las capacidades: origen social y elecciones educativas". Revista de la Asociación de Sociología de la Educación, 9 (1), 78-93. https:/ /ojs.uv.es/index.php/RASE/article/ view/8405/7998.

Van de Werfhorst, Herman G. y Andersen, Robert (2005): "Social Background, Credential Inflation and Educational Strategies”. Acta Sociológica, 48 (4) https://doi.org/10.1177/0001699305059945.

\section{Nota biográfica}

Mónica López Ramírez es Doctora en Ciencia Social con especialidad en Sociología por El Colegio de México. Obtuvo la Maestría en Sociología por la Universidad Autónoma Metropolitana unidad Azcapotzalco y la Licenciatura en Sociología en la misma institución. Realizó una estancia posdoctoral en el Centre Population et Développement (CEPED) Université Paris Descartes-IRD en París, Francia. En 2016 fue acreedora al Premio ANUIES en la categoría de mejor tesis de doctorado sobre educación superior. Actualmente es Investigadora Asociada del Instituto de Investigaciones Sobre la Universidad y la Educación (IISUE) de la UNAM y Candidata a Investigadora Nacional por el Sistema Nacional de Investigación. Sus áreas de investigación son la internacionalización de la educación superior, la movilidad internacional de estudiantes, las trayectorias escolares y desigualdades educativas. 